\title{
CDS UV Brightenings Explained by Quasi-separatrices and Bald Patches in an S-shape Active Region
}

\author{
B. Schmieder and P. Démoulin
}

Observatoire de Paris, 92195 Meudon, Cedex Principal, France

L. Fletcher

Lockheed Martin Solar and Astrophysics Lab., Org L9-41, B252, 2351 Hanover Street, Palo Alto CA 94306, US

M. C. López Fuentes and C. H. Mandrini

Instituto de Astronomía y Física del Espacio, IAFE, CC67, Suc 28, 1428 Buenos Aires, Argentina

H. E. Mason and P. R.Young

Department of Applied Mathematics and Theoretical Physics, Cambridge University, Silver Street, Cambridge CA, UK

N. Nitta

Lockheed Martin Solar and Astrophysics Lab., Org L9-41, B252, 2351 Hanover Street, Palo Alto CA 94306, US

Abstract. We present multi-instrument observations of AR 8048, made between June 3 and June 51997 as part of SoHO JOP033. This active region (AR) has a sigmoid-like global shape and undergoes transient brightenings through which the stored energy is released.

Using a magneto-hydrostatic model, we compute coronal magnetic field. The large-scale magnetic lines confirm the sigmoidal characteristics of the AR. The field lines most closely matching the hotter SoHO/CDS loops extend along the quasi-separatrix-layers (QSLs) of the coronal field. Transition region (TR) brightenings observed with SoHO/CDS can be associated with both QSL intersections with the photosphere, and places where separatrices corresponding to bald patches (BPs, sites where field lines are tangent to the photosphere) lie at the photospheric plane. There are suggestions that the element abundances measured in the TR may depend on the type of topological structure present. TR brightenings associated with QSLs have coronal abundances, while those associated with BP separatrices have abundances closer to photospheric values. 


\section{Introduction}

The Joint Observing Program 33 (JOP 33) was an observating campaign of the Solar and Heliospheric Observatory (SoHO) that ran between June 3 and June 5 , 1997 observing the AR 8048 (Fletcher et al. 2001). AR 8048 was a well-isolated region with a sigmoidal appearance (Figure 1). The SoHO group of instruments (mainly CDS and MDI), Yohkoh/SXT and ground based observatories (Bialków) provided us with plasma diagnostics, magnetic field measurements, and both high and low temperature images of AR 8048.

The CDS data include a number of lines from ions of $\mathrm{Mg}$ and $\mathrm{Ne}$, providing the opportunity to investigate the $\mathrm{Mg} / \mathrm{Ne}$ abundance ratio. $\mathrm{Mg}$ and $\mathrm{Ne}$ are examples of low and high first ionisation potential ions (FIPs of 7.6 and 21.6 $\mathrm{eV}$, respectively). It has been known for some time that the abundances of low FIP relative to high FIP ions is higher in the corona than in the photosphere (Meyer 1985a, b).

The brightness distribution at various temperatures and the element abundances are likely to be linked to the magnetic configuration, since the magnetic field determines the channels for plasma and energy transport. Motivated by this, we compute the coronal field from MDI magnetograms and study the magnetic topology, both loop shapes and the locations of critical structures such as "bald patches"(BP) and "quasi-separatrix layers" (QSLs), which are important for understanding magnetic reconnection. The relationship between magnetic topology and solar emission has been explored in various wavelength regimes, though little in the UV and EUV part of the spectrum. In this work we will continue in this vein, emphasizing the relationship of TR brightenings and magnetic topology. By reconstructing the magnetic environment of the AR we have been able to find a relationship between the high temperature coronal emission in the core of the AR and QSLs, and between TR brightenings and the intersection of QSLs or BP separatrices with the photosphere. We further find that the type of magnetic structure (i.e. a QSL or BP separatrix structure) may affect the derived chemical abundances in different locations in the solar atmosphere.

\section{Analysis and Results}

The highest temperature line in this CDS dataset is the line of Si XII at $520.6 \AA$ with a temperature of maximum ionisation fraction of $2 \mathrm{MK}$. This emission tends to occur at the same location as the SXT emission in the core of the AR. Emission from locations corresponding to the ends of the hot core loops is present in lines of around one million degrees (e.g. $\mathrm{Mg} \mathrm{IX}$ ). This is consistent with loops which are hotter at the apex and cooler at the ends.

In TR lines (e.g. Ne V, VI; Mg V, VI), brightenings are observed concentrated near the trailing polarity of the larger bipole. These brightenings fluctuate in intensity and are suggestive of small-scale activity. On June 3 , the emission in Ne VI 562.8 $\AA$ at 14:09:19 UT, consisted chiefly of five bright regions labeled A-E on Figure 2a. These regions were visible in Ne lines from IV to VI. In $\mathrm{Mg} \mathrm{V}$, Features $\mathrm{A}$ and $\mathrm{C}$ were visible; while Features $\mathrm{A}, \mathrm{C}$ and $\mathrm{E}$ were visible in $\mathrm{Mg}$ VI. On the next two days, June 4 and 5, only one feature (at the location corresponding to B) is present. Densities in the A, B, C, D, E features, 
measured with CDS line ratio diagnostics (Mg VII), are consistent with normal TR values and remain constant from pixel to pixel, within the errors. Features $\mathrm{A}, \mathrm{C}$ and $\mathrm{E}$ have $\mathrm{Mg} / \mathrm{Ne}$ abundance ratio as high as previously measured for coronal structures, while features B and D have a somewhat lower abundance ratio (but above typical photospheric value).

Following Démoulin et al. (1996), we have computed the locations of QSLs and analysed their relationship to the observed CDS TR brightenings. QSLs are regions where field lines initially close together separate widely when followed. QSLs are a generalization of the concept of separatrices to configurations without magnetic linkage discontinuities. Separatrices are present in the solar atmosphere in two cases, either when magnetic nulls appear in the configuration or when some field lines are tangentially touching the photosphere (these field lines are curved up at the photosphere). This latter case can happen along portions of the inversion line for the component of the field normal to the photosphere. These portions are called bald patches (BPs).

The coalignment of the CDS and Yohkoh data with MDI, together with the computation of the coronal magnetic field and its topology have shown the relationship between the emissions at various temperatures (TR and coronal) and the magnetic field topology (Figure 2b-d for June 3). At coronal temperatures, the Si XII emission, at the core of the AR, is related to the coronal $\mathrm{Mg}$ IX brightenings lying on QSLs. Field lines starting from these QSLs follow the shape of the Si XII emission. The most diffuse SXT emission is also related to magnetic lines linking QSLs at the main bipolar field.

Many features which are bright in the TR line of Ne VI lie close to the photospheric intersection of topological structures, both BP separatrices and QSLs. Features C, E and (possibly) A are found to be associated with QSLs. These TR brightenings have most probably their origin in the released of magnetic energy by reconnection at the QSLs, as described in the model of Démoulin and Priest (1997). Features B and D are rather found associated to BPs; in particular, feature D disappears on June 4 and 5 together with its BP and corresponding separatrices. Magnetic reconnection in a BP topology, not only releases energy, but allows the lifting-up of dense and cold material (e.g. Titov et al. 1993, Cheng and Choe 1998).

Therefore, the observed differences in the $\mathrm{Mg} / \mathrm{Ne}$ abundance ratio between features A, C, E and B, D are likely to be a direct consequence of magnetic topology involved.

\section{References}

Cheng, C. Z. \& Choe G. S. 1998, ApJ 505, 376

Démoulin P., Hénoux J.C., Priest E.R. and Mandrini C.H. 1996, A\&A 308, 643

Démoulin, P. \& Priest, E.R. 1997, Solar Phys. 175, 123

Fletcher, L., López-Fuentes, M.C., Mandrini, C.H., Schmieder, B., Démoulin, P., Mason, H., Young, P., Nitta, N. 2001, Solar Phys., submitted

Meyer, J.-P. 1985a, ApJS 57, 173

Meyer, J.-P. 1985b, ApJS 57, 151

Titov, V.S., Priest, E.R., Démoulin, P. 1993, A\&A 276, 564 

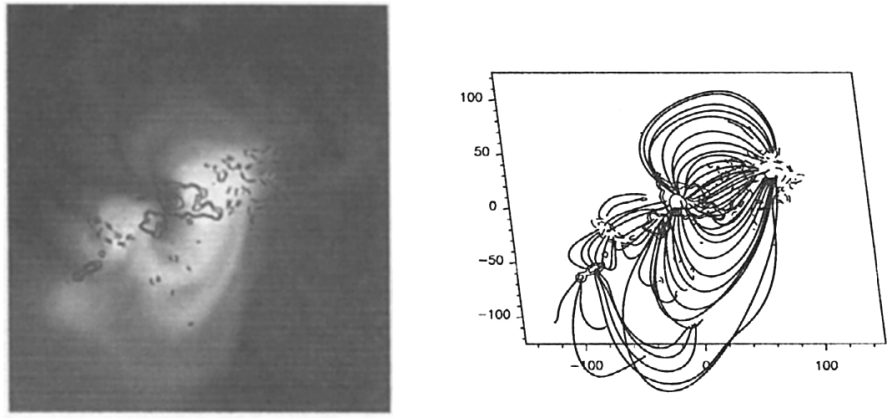

Figure 1. SXT images in Al12 filter on June 3 at 14:08 UT. The images have been overlaid to MDI magnetograms for comparison with the magnetic model (right). The isocontours of $B_{l}$ are $\pm 50,100$ and $500 \mathrm{G}$. A set of field lines has been added to be compared with SXT. The sizes of the frames are expressed in $\mathrm{Mm}$ and the shape is due to projection effects.
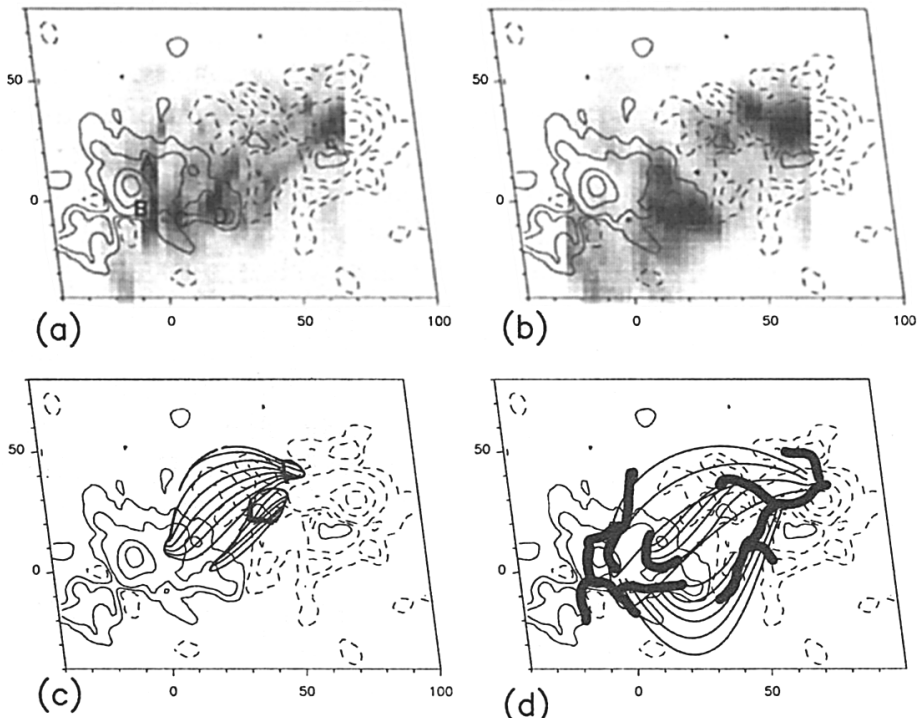

(d)

Figure 2. A comparison of the magnetic topology and CDS data for June 3. (a) and (b) are, respectively, an overlay of Ne VI and Mg IX (reversed images) observations at 14:09:39 UT with the MDI in Fig. 1. In this case the isocontours of $B_{l}$ are $\pm 20,100,250,500,1000 \mathrm{G}$. We have labeled the Ne VI features in (a). (c) shows the locations of BPs, and the intersection of their separatrices with the photosphere. BPs (separatrices) are drawn in continuous thick (thin) black lines, associated field lines have been added. (d) shows the intersection of QSLs (thick lines) with the photosphere, some field lines issued from QSLs are drawn. 